\title{
Geometric Picture for Coupled Electron-Nuclear Dynamics
}

\author{
VLADIMIR CHERNYAK, ${ }^{1}$ SHAUL MUKAMEL ${ }^{2}$ \\ ${ }^{1}$ Corning Incorporated, Process Engineering and Modeling, Corning, New York 14831 \\ ${ }^{2}$ Department of Chemistry, University of Rochester, Rochester, New York 14627
}

Received 15 August 2001; accepted 5 March 2002

DOI 10.1002/qua.949

\begin{abstract}
Equations of coupled electron-nuclear dynamics (CEND) that describe molecular dynamics within a fully classical approach have been derived. Utilization of the language of fiber bundles and connections (gauge fields) allows one to represent the resulting classical Hamilton equations in an invariant (coordinate-covariant) form. Applications of the CEND equations to calculations of off-resonant nonlinear molecular polarizabilities are outlined. The CEND equations are shown to resemble the END equations derived earlier once the Thouless representation of single Slater determinants is adopted. (c) 2002 Wiley Periodicals, Inc. Int J Quantum Chem 0: 1-13, 2002
\end{abstract}

\section{Key words:}

\section{Introduction}

B uilding effective numerical approaches for molecular dynamics simulations of large molecules constitutes one of the most challenging problems of theoretical and computational chemistry. The problem is of extreme complexity since it involves quantum dynamics of the vibrational and electronic degrees of freedom. The electronic problem alone is difficult since it involves manybody techniques for interacting electrons and constitutes an important area of quantum chemistry.

Correspondence to: S. Mukamel; e-mail: mukamel@chem. rochester.edu.

Contract grant sponsor: National Science Foundation.
On the other hand applying classical or semiclassical approximations (when possible) substantially reduces the numerical effort and makes it possible to perform numerical simulations in cases where the full quantum description has no chance of being handled. For example, the time-dependent HartreeFock (TDHF) approach [1] that can be considered as a classical counterpart of the full quantum manyelectron dynamics [2] combined with some powerful numerical diagonalization techniques [3] has demonstrated its capacity in modeling the resonant optical properties of large organic molecules [4]. However, even after adopting a classical approximation for the electronic degrees of freedom, following the nuclear dynamics on the quantum level is not practical except for very small molecules. On the other hand in certain cases, e.g., when we are 
interested in off-resonant optical properties of molecules, a fully classical approach captures all essential features of coupled electron-nuclear dynamics.

Such an approach where the vibrational degrees of freedom are treated fully classically and the electronic motions are accounted for within the TDHF scheme which makes the approach fully classical has been introduced [5] and bears the name electron-nuclear dynamics (END) approach. In this article we concentrate on geometrical issues of the END equations. By utilizing a variational principle in its invariant (i.e., coordinate-free) formulation we derive a set of classical dynamical equations hereafter referred to as coupled electron and nuclear dynamics (CEND) equations and demonstrate that they reduce to the END equations for a special parametrization of a Grassmanian manifold that represents the space of single Slater determinants, the key object of the TDHF scheme. This immediately implies that the CEND equations are equivalent to those of END. However, the invariant form allows for different parametrizations that might be more useful for certain applications. Another advantage of the invariant approach originates from the fact that the finite-dimensional space of the single electron states used in numerical calculations is generally speaking different for different molecular geometries. This implies that a natural language for describing the coupled dynamics in invariant terms is the language of vector fiber bundles and connections in them (or, using a more physical term, gauge fields). Transformations to different basis sets in the spaces of single-electron states that are moleculargeometry dependent can be considered gauge transformations.

The article is organized as follows. In Section II we present a brief overview of the Hamiltonian approach to the variational dynamics and connect the Poisson bracket to the corresponding symplectic structure. In Section III we present an approach to the classical coupled dynamics base on vector bundles and connections and introduce some canonical connections that are used in the approach. In Section IV we describe the basic dynamical structures, namely the Poisson bracket and the Hamiltonian, expressing them using connections in the relevant vector bundles. In Section $\mathrm{V}$ we derive the CEND equations based on the dynamical structures presented in Section IV. Finally, in Section VI we derive the dynamical equations for the so-called intermediate frequency limit that corresponds to the case of off-resonant molecular optical polarizabilities. These equations have been utilized to derive closed expressions for the vibrational contributions to off-resonant optical polarizabilities [6]. The basic concepts of fiber bundles and connections relevant for our approach are presented in the Appendices.

\section{Hamiltonian Formulation of the Time-Dependent Variational Principle}

Our analysis is based on an invariant representation for the CEND. As stated in the Introduction, the CEND equations are equivalent to those of the END [5] and can be viewed as the END equations represented in an invariant form, rather than using a specific choice of coordinates in the coupled electron-nuclear phase space. The invariant representation of the coupled dynamics has several advantages. The equations adopt a simple form and have a clear interpretation in terms of differential geometry. Closed dynamical equations for the parameters can be easily derived for arbitrary parametrization of the phase space. We are not restricted to the Thouless representation [1] and can use other representations which are more suitable for calculations of optical response functions.

Our approach is based on the Hamiltonian form of the time-dependent variational principle (TDVP) which can be formulated as follows. Let $\hat{H}$ be a quantum Hamiltonian which acts in the $L-$ dimensional complex Hilbert space of states $\mathbb{C}^{L}$. The space of normalized wavefunctions (where wavefunctions which differ by a phase factor are identical) is represented by the complex projective space $\mathbb{C P}^{L-1}$. Let $M \subset \mathbb{C P}^{L-1}$ be the space of trial normalized wavefunctions, defined up to a phase factor. Since the TDVP is based on the minimal-action principle, it yields classical Lagrangian dynamics on $M$. The action used in the TVDP only contains first order time derivatives and the conjugated momenta therefore become functions of coordinates; this can be considered as the appearance of the constraints that appear in the way of obtaining the Hamiltonian classical dynamics starting with its Lagrangian counterpart. As a result, the phase space of the corresponding Hamiltonian dynamics coincides with coordinate space of the Lagrangian dynamics and is represented by $M$. The Hamiltonian dynamics is determined by two structures: the classical Hamiltonian $H$ which is a function on $M$ and a binary operation that associates with two functions $f, g$ on $M$, the function $\{f, g\}$ called their Poisson bracket. The Poisson bracket satisfies the following 
properties $[7,8]$ :

$$
\begin{aligned}
& \{f, g\}=-\{g, f\} \\
& \{f, g h\}=\{f, g\} h+g\{f, h\} \\
& \{f,\{g, h\}+\{g,\{h, f\}+\{h,\{f, g\}\}\}\}=0 .
\end{aligned}
$$

Equation (2.2) is known as the differential property whereas Eq. (2.3) is the Jacobi identity Eqs. (2.1) and (2.2) imply that the Poisson bracket is determined by an antisymmetric bivector field $\hat{\omega}$. For some local coordinates $x_{k}$ on $M, \hat{\omega}$ can be represented in a form

$$
\hat{\omega}=\sum_{i k} \hat{\omega}_{i k}(x) \frac{\partial}{\partial x_{i}} \otimes \frac{\partial}{\partial x_{k}}
$$

with a $\hat{\omega}_{i k}=-\hat{\omega}_{k i}$, and the Poisson bracket adopts the form

$$
\{f, g\}=\sum_{i k} \hat{\omega}_{i k}(x) \frac{\partial f}{\partial x_{i}} \frac{\partial g}{\partial x_{k}} .
$$

Note that even though Eq. (2.5) represents the Poisson bracket using a system of local coordinates, the operation itself is independent of the choice of coordinate system since it is defined by the action of an invariant object, i.e., a bivector field on a pair of functions; the action itself is invariant as well. If the Poisson bracket is nondegenerate it describes a symplectic structure on $M[8,9]$, which is a 2 -form $\omega$ on $M$,

$$
\omega=\sum_{i k} \omega^{i k}(x) d x_{i} \wedge d x_{k}
$$

with $\omega^{i k}(x) \equiv[\hat{\omega}(x)]_{i k}^{-1}$; i.e., $\sum_{k} \omega^{i k}(x) \hat{\omega}_{k j}(x)=\delta_{j}^{i}$. Although Eq. (2.6) describes $\omega$ using a system of local coordinates, the form $\omega$ as well as the Poisson bracket $\hat{\omega}$ is an invariant object.

The Jacobi identity [Eq. (2.3)] has a clear interpretation in terms of the corresponding symplectic structure. The form $\omega$ should be closed; i.e., its exterior differential is zero $[8,9]$ :

$$
d \omega=\sum_{i k j} \frac{\partial \omega^{i k}(x)}{\partial x_{j}} d x_{j} \wedge d x_{i} \wedge d x_{k}=0 .
$$

We are now in a position to describe the classical Hamiltonian dynamics on the space of trial wavefunctions $M$. The Hamiltonian dynamics is obtained from the Lagrangian dynamics in a standard way $[7,8,10]$. The latter is obtained from the minimal action principle for the action of the TDVP. This yields the following structures on $M$ : The classical Hamiltonian $H(x)$ has the form

$$
H(x) \equiv\langle x|\hat{H}| x\rangle,
$$

where the immersion $M \subset \mathbb{C P}^{L-1}$ allows one to treat points in $M$ as wavefunctions. The sympletic structure $\omega_{M}$ is induced from the canonical symplectic structure $\omega_{L}$ on $\mathbb{C P}^{L-1}$ by the immersion $M \subset$ $\mathbb{C P}^{L-1}$,

$$
\omega_{M}=\sum_{i k} \sum_{m n} \omega_{L}^{m n}(s(x)) \frac{\partial s_{m}}{\partial x_{i}} \frac{\partial s_{n}}{\partial x_{k}} d x_{i} \wedge d x_{k},
$$

where

$$
\omega_{L}=\sum_{m n} \omega_{L}^{m n}(s) d s_{m} \wedge d s_{n}
$$

is the canonical symplectic structure on $\mathbb{C P}^{L-1}$ which can be described by the following properties: it is compatible with the complex analytical structure on $\mathbb{C P}^{L-1}$ and invariant with respect to unitary transformations of the Hilbert space. In Eqs. (2.9) and (2.10) $s_{M}$ denotes a set of local coordinates on $\mathbb{C P}^{L-1}$, and $s(x)$ describes the immersion $M \subset$ $\mathbb{C P}^{L-1}$.

\section{Classical Representation of the Coupled Electronic and Nuclear Dynamics}

In this section we develop an invariant representation for the coupled electronic and nuclear dynamics based on the Hamiltonian formulation of the TVDP. It has been pointed out [5] that understanding the coupled electron-nuclear dynamics requires the implementation of some basic consequences of the theories of Lie groups, generalized coherent states, and classical dynamics [9, 11, 12]. We shall demonstrate that the most natural language which provides insight onto the nature of the electron-nuclear coupling and allows one to interpret the dynamical equations in a simple and unambiguous way is the language of differential geometry including such structures as fiber bundles and connections in them [13-15]. To set the stage we start by introducing the model and the notation for the basic structures of our approach. The definitions and the basic properties using the formal language of differential geometry are summarized in Appendix A.

Let $M_{R}$ be the space of nuclear coordinates, hereafter referred to as the configuration space. Since the nuclei in a molecule may not occupy the same position, and an exchange of identical nuclei does not change the state of the system, $M_{R}$ is nontrivial homotopically and homologically has been the subject of numerous studies in algebraic topology. 
We next introduce the vector space $U$ of singleelectron orbitals. In the ab initio approach, $U$ is the Hilbert space of two-component (because of the spin) complex-valued functions of three coordinates in real space. To avoid difficulties connected with infinite-dimensional Hilbert spaces, we assume that $U$ is finite-dimensional. Its dimensionality $\operatorname{dim}(U)$ can be, however, very high. The space of $N$-electron states is represented by the $N$ th exterior power $\Lambda^{N}(U)$ which is generated by single Slater determinates $u_{1} \wedge u_{2} \wedge \cdots \wedge u_{N}$, where $u_{j}$ are vectors in $U$. The wavefunctions of the joint electron-nuclear system are represented by functions on the configurational space $M_{R}$ which take their values in $\Lambda^{N}(U)$. The quantum Hamiltonian $\hat{H}$ of the joint system can be represented using the electron annihilation (creation) operators $c_{j}\left(c_{j}^{+}\right)$where $|j\rangle$ represents some basis set in $U$

$$
\hat{H}=\hat{H}_{0}+\hat{H}_{1},
$$

where

$$
\hat{H}_{0} \equiv \sum_{\mu} \frac{P_{\mu}^{2}}{2 M_{\mu}}+u(\mathbf{r})
$$

is the nuclear Hamiltonian written in terms of the nuclear momentum (coordinate) operators $P_{\mu}\left(r_{\mu}\right)$ whereas the electronic Hamiltonian

$$
\hat{H}_{1} \equiv \sum_{i j} t_{i j}(\mathbf{r}) c_{i}^{+} c_{j}+\frac{1}{2} \sum_{i j r s} v_{i j, r s}(\mathbf{r}) c_{i}^{+} c_{j}^{+} c_{r} c_{s}
$$

includes the coupling between electronic and nuclear motions through the r-dependence of $t(\mathbf{r})$ and $v(\mathbf{r})$. For a fixed space of single-electron orbitals this dependence constitutes the only source of coupling between the electronic and nuclear motions. In practice, however, one always deals with a subspace $V \subset U$ of $U$ which describes the electron orbitals included in a calculation. In many cases, to avoid numerical divergences [5], the subspace of relevant orbitals should depend on the nuclear configuration, which yields a set of spaces $V(\mathbf{r}) \subset U$. Using the language of differential geometry, we have a fiber bundle, i.e., a set of spaces $V(\mathbf{r})$ (fibers) parametrized by the configuration space $M_{R}$ (the base). Strictly speaking, this parametrization should be smooth. This can be achieved by introducing the total space $E$ of the bundle together with the smooth projection map p: $E \rightarrow M_{R}$ so that the fiber $V(\mathbf{r})$ over $\mathbf{r} \in M_{R}$ is represented by $p^{-1}(\mathbf{r})$. As a set the total space $E$ constitutes simply a union of all fibers $E=\bigcup_{\mathbf{r} \in M_{R}} V(\mathbf{r})$. However, such a formulation provides parametrization with the smooth structure by requiring $E$ to be a smooth manifold whereas $p$ is a smooth map. In our case $E$ can be defined as a submanifold of $M_{R} \times U$ represented by pairs $(\mathbf{r}, u)$ with $u \in V(\mathbf{r})$. We have defined the basic bundle in our theory. All other bundles which appear in our theory can be obtained by using a clear and general construction of extending certain operations from spaces to bundles by applying them to fibers. For example, the operation of the $N$ th exterior power produces the vector space $\Lambda^{N}(V)$ out of a vector space $V$. Its extension to fiber bundles works in the following way: For a bundle $E$ over $M_{R}$, the bundle $\Lambda^{N}(E)$ over $M_{R}$ is defined as a set to fibers $\Lambda^{N}(V(\mathbf{r}))$ [we reiterate that $V(\mathbf{r})$ are the fibers of $E]$. The smooth structure on $\Lambda^{N}(E)$ is defined in a standard way [13-15] which will not be discussed here.

The total space of the bundle $\Lambda^{N}(E) \rightarrow M_{R}$ can be viewed as the space of the wavefunctions in the joint system of nuclei and $N$ electrons where only the relevant orbitals are taken into account. Another important bundle is obtained from $E$ using the extension of the construction End which associates with a sector space $V$ the space $\operatorname{End}(V)$ of linear transformations (endomorphisms) of $V$. Thus End $(E)$ constitutes a set of fibers $\operatorname{End}(V(\mathbf{r}))$ parametrized by $M_{R}$.

We conclude our introduction to the basic concepts of fiber bundles which will be used in our theory with the construction of a connection in a bundle. This construction naturally arises when one makes an attempt to define calculus in a bundle. A natural generalization of maps that is connected with bundles is represented by "maps" on $M_{R}$ whose values at any $\mathbf{r} \in M_{R}$ belong to $V(\mathbf{r})$. Strictly speaking, these are not normal maps since their values at different points belong to different spaces. They are called the sections of the bundle defined as follows: a section $s$ is a map $s: M_{R} \rightarrow E$ so that for any $\mathbf{r} \in M_{R}, p(s(\mathbf{r}))=\mathbf{r}$. An attempt to differentiate a section $s(\mathbf{r})$ faces the following difficulty: $s(\mathbf{r}+\delta \mathbf{r})$ and $s(\mathbf{r})$ belong to different spaces $V(\mathbf{r}+\delta \mathbf{r})$ and $V(\mathbf{r})$ even when $\delta \mathbf{r}$ is small. To evaluate the derivative of a section one needs to establish a connection between the spaces $V(\mathbf{r}+\delta \mathbf{r})$ and $V(\mathbf{r})$ for an infinitely small $\delta \mathbf{r}: s(\mathbf{r}+\delta \mathbf{r})$ can then be translated to $s^{\prime}(\mathbf{r}+\delta \mathbf{r}, \delta \mathbf{r}) \in V(\mathbf{r})$ and the derivative $\nabla_{\mu} s(\mathbf{r})$ is defined as $s^{\prime}(\mathbf{r}+\delta \mathbf{r}, \delta \mathbf{r})-s(\mathbf{r})=\sum_{\mu} \nabla_{\mu} s(\mathbf{r}) \delta \mathbf{r}_{\mu}$. Since there is no canonical way to do this translation, the differentiation involves an additional structure responsible for the translation, which is called a connection. The latter can be naturally introduced in the following way. With any point $e=(\mathbf{r}, v) \in E$ with $v \in V(\mathbf{r})$ we associate a vector subspace $\mathbb{T}_{e}(\nabla, E) \subset$ 
$\mathbb{T}_{e}(E)$ of the tangent space $\mathbb{T}_{e}(E)$ to $E$ at point $e$ with $\operatorname{dim} \mathbb{T}_{e}(\nabla, E)=\operatorname{dim} M_{R}$. The space $\mathbb{T}_{e}(\nabla, E)$ determines the aforementioned translation: starting with $v \in V(\mathbf{r})$ and $\delta \mathbf{r}$ we find the unique vector $\delta e \in$ $\mathbb{T}_{(\mathbf{r}, v)}(\nabla, E)$ with $p(\delta e)=\delta \mathbf{r}$ which yields $e+\delta e=$ $\left(\mathbf{r}+\delta \mathbf{r}, v^{\prime}\right)$ with $v^{\prime} \in V(\mathbf{r}+\delta \mathbf{r}) ; v^{\prime}$ constitutes the result of translation of $v$ along $\delta \mathbf{r}$. There is an equivalent straightforward way to introduce a connection as a rule of differentiation which associates with a section $s(\mathbf{r})$ its derivative $\nabla_{\mu} s(\mathbf{r})$ with the following property satisfied for any function $f(\mathbf{r})$ on $M_{R}$ :

$$
\nabla_{\mu}(f(\mathbf{r}) s(\mathbf{r}))=f(\mathbf{r}) \nabla_{\mu} s(\mathbf{r})+\partial_{\mu} f(\mathbf{r}) s(\mathbf{r}) .
$$

The equivalence of the two definitions is established by introducing a local basis set of sections $v_{1}(\mathbf{r}), \ldots, v_{N_{0}}(\mathbf{r})$ and introducing the coefficients $A_{i k, \mu}$ of the connection $\nabla$ in this basis set by

$$
\nabla_{\mu} v_{i}(\mathbf{r})=-\sum_{j} A_{j i, \mu}(\mathbf{r}) v_{j}(\mathbf{r}) .
$$

Introducing the coefficients $s_{j}(\mathbf{r})$ of a section $s(\mathbf{r})$,

$$
s(\mathbf{r})=\sum_{j} s_{j}(\mathbf{r}) v_{j}(\mathbf{r}),
$$

we obtain by applying Eq. (3.4) for $\nabla_{\mu} s_{j}(\mathbf{r})$

$$
\nabla_{\mu} s_{j}(\mathbf{r})=\partial_{\mu} s_{j}(\mathbf{r})-\sum_{k} A_{j k, \mu}(\mathbf{r}) s_{k}(\mathbf{r}) .
$$

In field theory, Eq. (3.7) is known as the definition of the "long" derivative which includes the gauge field $A_{\mu}$.

As stated above, for a tangent to $M_{R}$ at point $\mathbf{r}$ vector $\delta \mathbf{r} \in \mathbb{T}_{\mathbf{r}}\left(M_{R}\right)$ there is one and only one vector $\delta e \in \mathbb{T}_{(\mathbf{r}, v)}(\nabla, E)$ so that $p(\delta e)=\delta \mathbf{r}$. This defines a set of lifting maps $L_{(\mathbf{r}, v)} \mathbb{T}_{\mathbf{r}}\left(M_{R}\right) \rightarrow \mathbb{T}_{(\mathbf{r}, v)}(E)$. These maps uniquely describe the connection as the set of subspaces $\mathbb{T}_{(\mathbf{r}, v)}(\nabla, E)$ which are given by $\mathbb{T}_{(\mathbf{r}, v)}(\nabla, E)=$ $i m L_{(\mathbf{r}, v)}$. Using the basis set of sections $v_{j}(\mathbf{r})$ we can characterize points $(\mathbf{r}, v)$ in $E$ by pairs $(\mathbf{r}, s)$ where $s$ is a vector set of numbers $s_{j}$ using the expansion $v=\sum_{j} s_{j} v_{j}(\mathbf{r})$. Defining the lifting maps by

$$
L_{(\mathbf{r}, s)}(\delta \mathbf{r})=\delta \mathbf{r} \oplus \sum_{\mu} \delta r_{\mu} A_{\mu}(\mathbf{r}) S
$$

we can interpret Eq. (3.7) as the derivative which involves the translation of a section along $\mathbb{T}_{(\mathbf{r}, v)}(\nabla, E)$. This establishes a connection between two equivalent definitions of a connection: for a given basis set, a matrix $A_{\mu}(\mathbf{r})$ determines the rule of differentiation on the one hand and describes the along-the-fiber component of the translation between two infinitely close fibers.
These definitions make it possible to describe the phase space of the classical coupled electronnuclear dynamics based on the TDVP. In the case of a fixed space of single-electron orbitals $U$ we follow Ref. [5] and choose the space of trial wavefunctions to be represented by direct products of a coherent state of the nuclei and a coherent state of electrons, i.e., a single Slater determinant. To obtain the same dynamics as in Ref. [5] and to avoid difficulties in future applications when restricting the basis to relevant orbitals, we set the Planck constant in the nuclear space $\hbar=0$. This makes the Gaussian wavepackets representing the nuclear coherent states infinitely sharp. The phase space, i.e., the space of trial wavefunctions, has the form of a direct product $M_{0} \times M_{1}$, of nuclear $M_{0}$ and electronic $M_{1}$ components. $M_{0}$ is represented by the set of nuclear positions and momenta and for the sake of generality will be considered as a bundle $M_{0} \stackrel{P_{0}}{\rightarrow} M_{R}$ over $M_{R}$ whose fibers are the spaces of nuclear momenta. $M_{1}$ is naturally given by the Grassman manifold $G_{N}(U)$ of all $N$-dimensional vector subspaces of $U$. According to [14] $G_{N}(U)$ can be considered as a subspace $G_{N}(U) \subset \operatorname{End}(U)$ of linear operators $\rho$ in $U$ which satisfy the following restrictions: $\rho^{2}=\rho$, $\rho^{+}=\rho$, and rank $\rho=N$. Using this language, $\rho$ has a meaning of the single-electron density matrix calculated on a single Slater determinant. We can now restrict ourselves to the relevant orbitals by requiring that for a nuclear coherent state $(\mathbf{P}, \mathbf{r})$ the electronic single Slater determinant should be constructed out of the orbitals which belong to $V(\mathbf{r})$. Note that this construction is possible because of the infinitely narrow width of the nuclear coherent states. Otherwise the Slater determinants for different $\mathbf{r}$ belonging to the region where the Gaussian wavepacket is concentrated would be based on different spaces $V(\mathbf{r})$. The restricted phase-space $M$ has a natural structure of a bundle. Since for $\mathbf{r} \in M_{R}$ the space of relevant single Slater determinants is given by $\left(G_{N}(V(\mathbf{r}))\right.$, the joint space of nuclear positions and electronic degrees of freedom is given by extending the construction of forming Grassmanian out of a vector space to the bundle $p: E \rightarrow M_{R}$ which yields the Grassmanian $G(p): G_{N}(E) \rightarrow M_{R}$. Similar to the vector space $U$ we have for the bundles $G_{N}(E) \subset \operatorname{End}(E)$. To obtain the full phase space we need to define a bundle over $M_{R}$ with the fibers being the cartesian products of the spaces of momenta and $\left(G_{N}(V(\mathbf{r}))\right.$. This is achieved by extending the construction of the cartesian products to the bundles: $M \equiv M_{0} \times_{M_{R}} G_{N}(E)$. The points of $M$ are represented by the triples $(\mathbf{r}, \mathbf{P}, \rho)$ with $\rho$ being a lin- 
ear operator in $V(\mathbf{r})$ with $\rho^{2}=\rho, \rho^{+}=\rho$, and rank $\rho=N$. Note that we have two natural immersions: $M \subset M_{0} \times_{M_{R}}$ End $(E)$ and $M \subset M_{0} \times M_{1}$.

Finally we note that although bundles do not have canonical connections in them, the immersion $E \in M_{R} \times U$ of our bundle $E$ provides a natural connection in it. As a way of differentiation of sections it is defined as follows. A section $s(\mathbf{r})$ of $E$ can be considered as a function $s: M_{R} \rightarrow U$. Its derivative $\partial_{\mu} s(\mathbf{r})$ is a function but not a section of $E$ since $\partial_{\mu} s(\mathbf{r})$ does not, in general, belong to $V(\mathbf{r})$. We define $\nabla_{\mu} s(\mathbf{r})$ as the projection of $\partial_{\mu} s(\mathbf{r})$ on $V(\mathbf{r})$ which yields a connection $\nabla$ in $E$. The same connection can be defined alternatively as a set $\mathbb{T}_{(\mathbf{r}, v)}(\nabla, E)$ as follows. Denoting by $V(\mathbf{r})^{\perp}$ the orthogonal to $V(\mathbf{r})$ component in $U$ we have the immersions $\mathbb{T}(\mathbf{r})(M) \oplus V(\mathbf{r})^{\perp} \subset$ $\mathbb{T}_{(\mathbf{r}, v)}\left(M_{R} \times U\right)$ and $\mathbb{T}_{(\mathbf{r}, v)}(E) \subset \mathbb{T}_{(\mathbf{r}, v)}\left(M_{R} \times U\right)$. We then define $\mathbb{T}_{(\mathbf{r}, v)}(\nabla, E) \equiv\left(\mathbb{T}_{\mathbf{r}}(M) \oplus V(\mathbf{r})^{\perp}\right) \cup \mathbb{T}_{(\mathbf{r}, v)}(E)$. The meaning of this connection is that the translation from $V(\mathbf{r})$ to $V(\mathbf{r}+\delta \mathbf{r})$ goes in the direction orthogonal to $V(\mathbf{r})$.

In summary, we have $M_{0} \rightarrow M_{R}$ which can be considered as a cotangent bundle on $M_{R}$ (since the momenta are linear functionals on tangent vectors). We have a vector bundle $E \rightarrow M_{R}$ with a connection $\nabla$ in it. The phase space $M$ of the joint dynamics is given by

$$
M=M_{0} \times_{M_{R}} G_{N}(E) .
$$

In the next section we derive the classical Hamiltonian and the Poisson bracket on $M$ which will be described in terms of the connection $\nabla$.

\section{Coupled Electronic and Nuclear Dynamics: Classical Hamiltonian and Poisson Bracket}

In the previous section we have identified the phase space $M$ of the complex classical dynamics as a bundle over the configuration space $M_{R}$. We have also introduced the basic structures which will be involved in the dynamics: a vector bundle $E$ representing the single orbital spaces and a connection $\nabla$ in it. In this section we derive the classical Hamiltonian and Poisson bracket in $M$ which constitute the basic ingredients of the dynamics and express them in terms of the basic structures. This will be done in the following way: We start by deriving the Hamiltonian and the symplectic structure in the extended phase space $M_{0} \times M_{1}$ which is based on the full set $U$ of electron orbitals. We then derive the Hamiltonian and symplectic structure in $M$, inducing them from $M_{0} \times M_{1}$ by implementing the immersion $M \subset M_{0} \times M_{1}$. Finally we obtain the Poisson bracket in $M$ out of the symplectic structure by implementing the standard procedure outlined in Section II.

For our ansatz, a wavefunction is a direct product of an electronic wavefunction and an infinitely narrow nuclear wavepacket. The classical Hamiltonian $H(\mathbf{r}, \mathbf{P}, \rho)$ which represents the expectation value of the quantum Hamiltonian [Eqs. (3.1) and (3.2)] then adopts the form

$$
H(\mathbf{r}, \mathbf{P}, \rho)=H_{0}(\mathbf{r}, \mathbf{P})+H_{1}(\mathbf{r}, \rho)
$$

with the nuclear Hamiltonian

$$
H_{0}(\mathbf{r}, \mathbf{P})=\sum_{\mu} \frac{P_{\mu}^{2}}{2 M_{\mu}}+u(\mathbf{r}) .
$$

$H_{1}$ can be considered a purely electronic Hamiltonian which depends on $\mathbf{r}$ parametrically. Following [6] it can be represented in the form

$$
H_{1}(\mathbf{r}, \rho)=\operatorname{Tr}(t(\mathbf{r}) \rho)+\operatorname{Tr}(\rho V(\mathbf{r}) \rho)
$$

where $t(\mathbf{r}) \in \operatorname{End}(U)$ are linear operators in $U$ represented by the matrix elements $t_{i j}(\mathbf{r})$ in Eq. (3.3). $V(\mathbf{r}) \in \operatorname{End}(\operatorname{End}(U))$ are superoperators acting in the space End $(U)$ of usual single-electron operators. The expression for $V$ in terms of the matrix elements $v_{i j, r s}$ of Eq. (3.3) is given in Ref. [6].

The Hamiltonian $H_{T}(\tau)$ of the system driven by an optical field $E(t)$ adopts the form

$$
H_{T}(\tau)=H-\mathcal{E}(\tau) \mathcal{P},
$$

with

$$
\mathcal{P}(\mathbf{r}, \rho)=\mu_{N}(\mathbf{r})+\operatorname{Tr}\{\rho \mu(\mathbf{r})\},
$$

where $\mu(\mathbf{r}) \in \operatorname{End}(U)$.

Since the trial wavefunctions are direct products of nuclear and electronic components, the symplectic structure $\omega$ on $M_{0} \times M_{1}$ is given by a sum of its nuclear and electronic components:

$$
\omega=\omega_{0}+\omega_{1} .
$$

The nuclear part has a standard symplectic-structure form for a set of coordinates and conjugated momenta:

$$
\omega_{0}=\sum_{\mu} d P_{\mu} \wedge d r_{\mu} .
$$

As stated in Section II, the component $\omega_{1}$ can be obtained by inducing it from the standard structure on the projective space representing all $\mathrm{N}$-electron states with respect to the canonical immersion 
$G_{N}(U) \subset \mathbb{C P}^{L_{0}}$ of the space of single Slater determinants into the space of all $\mathrm{N}$-electron states. In complex analytical geometry this immersion of a Grassmanian is known as the canonical embedding whereas the induced, symplectic structure is called the canonical Kähler structure on the Grassmanian [16]. Since $G_{N}(U)$ represents a set of coherent states $\omega_{1}$ can be represented in algebraic terms; the corresponding Poisson bracket has been described using the algebraic language in [6]. Let $\rho: G_{N}(U) \rightarrow \operatorname{End}(U)$ be the immersion described in Section III. We then have

$$
\omega_{1}=i \operatorname{Tr}\{\rho[d \rho \wedge d \rho]\} .
$$

Equation (4.8) can be explained as follows: $d \rho$ is a 1form with the values in $\operatorname{End}(U) ; d \rho \wedge d \rho$ is a 2 -form valued in $\operatorname{End}(U) \otimes \operatorname{End}(U)$. Applying the commutator followed by multiplying with $\rho$ we get the form $\rho[d \rho \wedge d \rho]$ valued in $\operatorname{End}(U)$. Finally, taking the trace yields the usual 2-form. If $y_{\mu}$ is a set of local coordinates in $G_{N}(U)$ we can recast Eq. (4.8) in the form

$$
\omega_{1}=i \sum_{\mu \nu} \operatorname{Tr}\left\{\rho(y)\left[\frac{\partial \rho}{\partial y_{\mu}}, \frac{\partial \rho}{\partial y_{\nu}}\right]\right\} d y_{\mu} \wedge d y_{\nu} .
$$

Equations (4.1)-(4.8) fully determine classical dynamics in $M_{0} \times M_{1}$. We are now left with two technical problems: to confine the classical Hamiltonian and symplectic structure onto $M \subset M_{0} \times M_{1}$ and to obtain the Poisson bracket $\hat{\omega}_{M}$ from the symplectic structure $\omega_{M}$.

Confining the Hamiltonian to $M \subset M_{0} \times M_{1}$ is a straightforward task which involves just projecting the operators $t(\mathbf{r})$ and $\mu(\mathbf{r})$ as well as the superoperators $V(\mathbf{r})$ from $U$ to $V(\mathbf{r}) \subset U$ at any point $\mathbf{r}$. The Hamiltonian on $M$ preserves the form given by Eqs. (4.1)-(4.5) with the only difference being that $t(\mathbf{r})$ and $\mu(\mathbf{r})$ should be considered as sections of the bundle $\operatorname{End}(E)$, where is $V(\mathbf{r})$ becomes a section of the bundle End $(\operatorname{End}(E))$. Confining the symplectic structure to $M \subset M_{0} \times M_{1}$ becomes a simple procedure once the language of bundles and connections is implemented.

So far we have put much effort in introducing the language of bundles and connections without much visible gain except for some interpretation of the phase space $M$ as a fiber bundle. At this point we can use this formal work to derive results virtually without calculations and obtain simple and clear interpretation.

To obtain the 2-form $\omega_{M}$ on $M$ we note that at any point $x=(\mathbf{r}, \mathbf{P}, \rho)$ it is given by an antisymmetric bilinear form acting in the tangent space $\mathbb{T}_{x}(M) \subset$ $\mathbb{T}_{x}\left(M_{0} \times M_{1}\right)$ which is determined by confining the form $\omega$ which acts in $\mathbb{T}_{x}\left(M_{0} \times M_{1}\right)$. Since in our case the momenta are decoupled from coordinates (i.e., the tangent bundle of $M_{R}$ is trivial) we have a natural decomposition $\mathbb{T}_{x}(M)=\mathbb{T}_{\mathbf{r}}^{*}\left(M_{R}\right) \oplus \mathbb{T}_{(\mathbf{r}, \rho)}\left(G_{N}(E)\right)$, where the first component is the space tangent to the momenta which can be identified with space of the momenta itself, the latter being the vector space dual to the tangent space [8]. The second component has a natural subspace $\mathbb{T}_{\rho}\left(G_{N}(V(\mathbf{r}))\right.$, which constitutes the subspace tangent to the fiber. The other subspace which allows to one partition $\mathbb{T}_{(\mathbf{r}, \rho)}\left(G_{N}(E)\right)$ into a direct sum is provided by the connection $\nabla$ in $G_{N}(E)$ induced by the connection $\nabla$ in $E$. The connection $\nabla$ can first be extended from $E$ to $\operatorname{End}(E)$ by requiring for any sections $\xi(\mathbf{r})$ and $s(\mathbf{r})$ of $\operatorname{End}(E)$ and $E$, respectively,

$$
\nabla_{\mu}(\xi(\mathbf{r}) s(\mathbf{r}))=\left(\nabla_{\mu} \xi(\mathbf{r})\right) s(\mathbf{r})+\xi(\mathbf{r})\left(\nabla_{\mu} s(\mathbf{r})\right) .
$$

Since the connection $\nabla$ in $\operatorname{End}(E)$ in the sense of a set of lifting maps is compatible with the constraints $\rho^{2}=\rho, \rho^{+}=\rho$, and rank $\rho=N$ it can be confined to $G_{N}(E)$ which yields the set of vector subspaces $\mathbb{T}_{(\mathbf{r}, p)}\left(\nabla, G_{N}(E)\right) \subset \mathbb{T}_{(\mathbf{r}, p)}\left(G_{N}(E)\right)$. Identifying these subspaces with $\mathbb{T}_{\mathbf{r}}\left(M_{R}\right)$ by means of the projection map $G_{N}(p)$ we arrive at a decomposition:

$$
\mathbb{T}_{x}(M)=\mathbb{T}_{\mathbf{r}}^{*}\left(M_{R}\right) \oplus \mathbb{T}_{\mathbf{r}}\left(M_{R}\right) \oplus \mathbb{T}_{\rho}\left(G_{N}(V(\mathbf{r}))\right) .
$$

The sum of the first two terms is hereafter referred to as the nuclear component, and the last term is the electronic component. The form $\omega_{M}$ has an important property which considerably simplifies the picture: $\omega_{M}(u \otimes v) \neq 0$ only if both $u$ and $v$ belong to the nuclear or electronic component. This can be rationalized as follows. The translation along the connection considered in the extended space $\mathbb{T}_{x}\left(M_{0} \times M_{1}\right)$ involves a shift $\delta \rho^{(0)}$ which may generate a cross term between $\mathbb{T}_{\mathbf{r}}\left(M_{R}\right)$ and the electronic component. However, according to the definition of the connection $\nabla$ in $E$ [see end of Section III before Eq. (3.9)] the shift of vectors goes in the directions orthogonal to $V(\mathbf{r})$. This implies that the shift $\delta \rho^{(0)}$ along the corresponding connection in $\operatorname{End}(E)$ involves the matrix elements between $V(\mathbf{r})$ and $V(\mathbf{r})^{+}$ only. $\delta \rho^{(1)}$ which is related to the electronic component acts in $V(\mathbf{r})$; the same is true for $\rho$ as well. This implies that $\operatorname{Tr}\left\{\rho\left[\delta \rho^{(1)}, \delta \rho^{(0)}\right]\right\}=0$ and the statement follows from Eq. (4.8). $\omega_{M}$ is thus decomposed into the nuclear and electronic part,

$$
\omega_{M}=\omega_{M}^{(0)}+\omega_{M}^{(1)}
$$

where $\omega_{M}^{(1)}$ is obviously given by Eq. (4.8) with $V(\mathbf{r})$ substituted instead of $U$. The component $\omega_{M}^{(0)}$ re- 
quires a minor calculation presented in Appendix B which yields

$$
\begin{aligned}
& \omega_{M}^{(0)}\left(\frac{\partial}{\partial P_{\mu}} \otimes \frac{\partial}{\partial r_{v}}\right)=\delta_{\mu \nu}, \\
& \omega_{M}^{(0)}\left(\frac{\partial}{\partial r_{\mu}} \otimes \frac{\partial}{\partial r_{\nu}}\right)=\operatorname{Tr}\left\{\rho F_{\mu \nu}(\mathbf{r})\right\} .
\end{aligned}
$$

The section $F_{\mu \nu}(\mathbf{r})$ of $\operatorname{End}(E)$ is the curvature of the connection $\nabla$ or in other words the intensity of the gauge field (see Appendix B for details) defined as

$$
\left[\nabla_{\mu}, \nabla_{\nu}\right] s(\mathbf{r})=F_{\mu \nu}(\mathbf{r}) s(\mathbf{r}) .
$$

Equations (4.12) and (4.13) determine the symplectic structure in $M$.

The Poisson bracket in the differential form, i.e., as a bivector field $\hat{\omega}_{M}$ on $M$, is obtained by inverting the matrix $\omega_{M}$ [see Eq. (2.6)]. Because of the absence of cross terms, this can be done in the nuclear and electronic spaces alone, which yields

$$
\hat{\omega}_{M}=\hat{\omega}_{M}^{(0)}+\hat{\omega}_{M}^{(1)}
$$

where the electronic contribution $\hat{\omega}_{M}^{(1)}$ is tangent to the fibers and is represented by the canonical Poisson brackets in the fibers $G_{N}(V(\mathbf{r}))$. The nuclear contribution has the form

$$
\begin{aligned}
\hat{\omega}_{M}^{(0)}(x)= & \sum_{\mu} \frac{\partial}{\partial P_{\mu}} \otimes L_{(\mathbf{r}, \rho)}\left(\frac{\partial}{\partial r_{\mu}}\right) \\
& -\sum_{\mu} L_{(\mathbf{r}, \rho)}\left(\frac{\partial}{\partial r_{\mu}}\right) \otimes \frac{\partial}{\partial P_{\mu}} \\
& +\sum_{\mu \nu} \operatorname{Tr}\left\{\rho F_{\mu \nu}(\mathbf{r})\right\} \frac{\partial}{\partial P_{\mu}} \otimes \frac{\partial}{\partial P_{\nu}}
\end{aligned}
$$

where $L_{(\mathbf{r}, \rho)}: \mathbb{T}_{\mathbf{r}}\left(M_{R}\right) \rightarrow \mathbb{T}_{(\mathbf{r}, \rho)}\left(G_{N}(E)\right)$ are lifting maps.

Equations (4.15) and (4.16) together with Eqs. (4.1) and (4.5) define the Poisson bracket and the Hamiltonian in the phase space $M$ and therefore fully determine the dynamics. Equations (4.15) and (4.16) also allow one to represent the Poisson bracket in an algebraic form, i.e., by evaluating it for a certain set of generating functions. It has been demonstrated [6] that if the phase space represents a set of generalized coherent states, there is a finite subspace of functions on it which generates the whole function algebra and is closed with respect to the Poisson bracket. In our case, one can find an infinite-dimensional subspace which satisfies the aforementioned properties. In spite of its infinite dimension it is suitable for all dynamical applications. This space is parametrized by a direct sum of spaces of functions $f(\mathbf{r})$ on $M_{R}$, vector fields $\beta(\mathbf{r})$ on $M_{R}$, and sections $\xi(\mathbf{r})$ of the bundle End $(E)$. The value of the function $f \oplus \alpha \oplus \xi$ at point $(\mathbf{r}, \mathbf{P}, \rho)$ is defined by

$$
(f \oplus \alpha \oplus \xi)(\mathbf{r}, \mathbf{P}, \rho) \equiv f(\mathbf{r})+P(\alpha(\mathbf{r}))+\operatorname{Tr}\{\rho \xi(\mathbf{r})\}
$$

where we have used the fact that $\alpha(\mathbf{r})$ is a vector in $M_{R}$ whereas $P$ is a linear functional on the space of vectors at point $\mathbf{r}$. The Poisson bracket of the functions of this type is immediately evaluated by substituting Eqs. (4.15) and (4.16) into Eq. (2.5), which yields

$$
\begin{aligned}
& \left\{f_{1} \oplus \alpha_{1} \oplus \xi_{1}, f_{2} \oplus \alpha_{2} \oplus \xi_{2}\right\} \\
& =\left(\alpha_{1}\left(f_{2}\right)-\alpha_{2}\left(f_{1}\right)\right) \oplus\left[\alpha_{1} \alpha_{2}\right] \\
& \quad \oplus\left(i\left[\xi_{1}, \xi_{2}\right]+\nabla \xi_{2}\left(\alpha_{1}\right)-\nabla \xi_{1}\left(\alpha_{2}\right)+F\left(\alpha_{1} \otimes \alpha_{2}\right)\right) .
\end{aligned}
$$

Here $\alpha(f)$ is the result of the action of the vector field $\alpha$ considered as a first-order differential operator on the function $f,\left[\alpha_{1}, \alpha_{2}\right]$ is the commutator of vector fields, $\nabla \xi(\alpha)$ denotes the value of the form $\nabla \xi \equiv$ $\sum_{\mu} \nabla_{\mu} \xi d r_{\mu}$ on the vector field $\alpha$, whereas $F\left(\alpha_{1} \otimes \alpha_{2}\right)$ stands for the value of the 2-form $F \equiv \sum_{\mu \nu} F_{\mu \nu} d r_{\mu} \wedge$ $d r_{v}$ on $\alpha_{1} \otimes \alpha_{2}$.

Dynamical equations which use certain coordinate systems in the phase space $M$ can be obtained by considering coordinates as functions on $M$. To obtain dynamical equations in this way one needs to derive the Poisson bracket between the coordinates treated as a function on $M$. We shall now introduce a coordinate system in $M$ which extends the coordinate system in the electronic space defined in [6] to the case of coupled dynamics and evaluate the Poisson bracket of the coordinates. This system is more convenient for calculating the optical susceptibilities than the Thouless representation and is based on the expansion which is valid in a vicinity of any point $\bar{\rho}$ in a Grassmanian [6]

$$
\rho=\bar{\rho}+\xi+T(\xi)
$$

where $\xi$ involves only the particle-hole components, i.e., $[\bar{\rho},[\bar{\rho}, \xi]]=\xi$, whereas $T(\xi)$ is represented by intraband components, i.e., $[\bar{\rho}, T(\xi)]=0$. Different forms for $T(\xi)$ are given in Refs. [4, 6, 14].

To obtain a system of local coordinates in an invariant form, i.e., without involving a basis set $v_{i}(\mathbf{r})$ of sections in $E$, we introduce a section $\bar{\rho}(\mathbf{r})$ of the bundle $G_{N}(E)$ and a set of sections $\xi_{\alpha}(\mathbf{r})$ of the bundle $\operatorname{End}(E)$ with the following properties. $\xi_{\alpha}(\mathbf{r})$ are particle-hole, i.e., $\left[\bar{\rho}(\mathbf{r}),\left[\bar{\rho}(\mathbf{r}), \xi_{\alpha}(\mathbf{r})\right]\right]=0, \xi_{\alpha}(\mathbf{r})$ come in pairs, i.e., $\xi_{-\alpha}(\mathbf{r})=\xi_{\alpha}^{+}(\mathbf{r})$, they constitute complete basis sets in the particle-hole components of 
End $(V(\mathbf{r}))$, and they are orthogonal in the following sense:

$$
\operatorname{Tr}\left\{\rho(\mathbf{r})\left[\xi_{\alpha}(\mathbf{r}), \xi_{\beta}^{+}(\mathbf{r})\right]\right\}=-\operatorname{sgn}(\alpha) \delta_{\alpha \beta} .
$$

It has been demonstrated [6] that all of these properties are satisfied for the eigenmodes of the TDHF equation. We can now parametrize the points $(\mathbf{r}, \mathbf{P}, \rho) \in M$ by a set of coordinates $\left(r_{\mu}, P_{\mu}, z_{\mu}\right)$ using the decomposition:

$$
\rho=\bar{\rho}(\mathbf{r})+\sum_{\alpha} z_{\alpha} \xi_{\alpha}(\mathbf{r})+T\left(\sum_{\alpha} z_{\alpha} \xi_{\alpha}(\mathbf{r})\right)
$$

with $z_{\alpha}=z_{\alpha}^{*}$.

Combining Eqs. (4.20) and (4.21) (see [6] for details) yields the following representation for the electron coordinates $z_{\alpha}$ :

$$
z_{\alpha}=\operatorname{sgn}(\alpha) \operatorname{Tr}\left\{\rho\left[\bar{\rho}(\mathbf{r}), \xi_{\alpha}^{+}(\mathbf{r})\right]\right\} .
$$

Equation (4.22) implies that $z_{\alpha}$ as functions on $M$ belong to the class described by Eq. (4.17), namely by the third component. The section of End $(E)$ which represents the function $z_{\alpha}$ is given by $\operatorname{sgn}(\alpha)\left[\bar{\rho}(\mathbf{r}), \xi_{\alpha}^{+}(\mathbf{r})\right]$ which is obtained by comparison of Eq. (4.17) and Eq. (4.22). The coordinates $P_{\mu}$ treated as functions on $M$ belong to the class given by Eq. (4.17) and are represented by vector fields $\partial / \partial r_{\mu}$. The Poisson bracket of the coordinates is therefore obtained by applying Eq. (4.18), which yields

$$
\begin{aligned}
\left\{P_{\mu}, f(\mathbf{r})\right\}= & \partial_{\mu} f(\mathbf{r}) \\
\left\{P_{\mu}, P_{\nu}\right\}= & \operatorname{Tr}\left\{\bar{\rho}(\mathbf{r}) F_{\mu \nu}(\mathbf{r})\right\}+\sum_{\alpha} \operatorname{Tr}\left\{\xi_{\alpha}(\mathbf{r}) F_{\mu \nu}(\mathbf{r})\right\} z_{\alpha} \\
& +\operatorname{Tr}\left\{T\left(\sum_{\alpha} z_{\alpha} \xi_{\alpha}(\mathbf{r}) F_{\mu \nu}(\mathbf{r})\right)\right\} \\
\left\{P_{\mu}, z_{\alpha}\right\}= & \operatorname{sgn}(\alpha) \operatorname{Tr}\left\{\bar{\rho}(\mathbf{r})\left[\nabla_{\mu} \bar{\rho}(\mathbf{r}), \xi_{\alpha}^{+}(\mathbf{r})\right]\right\} \\
& +\operatorname{sgn}(\alpha) \sum_{\beta} z_{\beta} \operatorname{Tr}\left\{\bar{\rho}(\mathbf{r})\left[\nabla_{\mu} \xi_{\alpha}^{+}(\mathbf{r}), \xi_{\beta}(\mathbf{r})\right]\right\} \\
& +\operatorname{sgn}(\alpha) \operatorname{Tr}\left\{T\left(\sum_{\beta} z_{\beta} \xi_{\beta}(\mathbf{r})\right)\right. \\
& \left.\times\left[\nabla_{\mu} \bar{\rho}(\mathbf{r}), \xi_{\beta}^{+}(\mathbf{r})\right]\right\} \\
\left\{z_{\alpha}, z_{\beta}\right\}= & \operatorname{sgn}(\alpha) \delta_{\alpha,-\beta} \\
& +\operatorname{sgn}(\alpha) \operatorname{sgn}(\beta) \operatorname{Tr}\left\{T\left(\sum_{\nu} z_{\nu} \xi_{\nu}(\mathbf{r})\right)\right. \\
& \left.\times\left[\bar{\rho}(\mathbf{r}), \xi_{\alpha}^{+}(\mathbf{r})\right],\left[\bar{\rho}(\mathbf{r}), \xi_{\beta}^{+}(\mathbf{r})\right]\right\} .
\end{aligned}
$$

\section{Equations of Motion for the Coupled Dynamics}

Equations of motion for the coupled dynamics can be obtained using the Hamiltonian given by Eqs. (4.1) and (4.5) and the Poisson bracket $\hat{\omega}_{M}$ in the differential form [Eqs. (4.15) and (4.16)]. The Hamiltonian equation of motion for $x=(\mathbf{r}, \mathbf{P}, \rho) \in M$ can be represented in the general form $[8,10]$

$$
\frac{d x}{d \tau}=\left(d H \otimes i_{\mathbb{T}_{x}(M)}\right) \hat{\omega}_{M}(x)
$$

which should be understood in the following way. The differential $d H$ of the Hamiltonian $H$, the latter being a function on $M$, constitutes a 1-form on $M$ which yields a number acting on a vector. Acting with $d H$ on the left component of the bivector $\hat{\omega}_{M}(x) \in \mathbb{T}_{x}(M) \otimes \mathbb{T}_{x}(M)$ yields a tangent vector, i.e., an element of $\mathbb{T}_{x}(M)$, which originates from the second component of the tensor product. This tangent vector in the right-hand side (r.h.s.) of Eq. (5.1) determines the value of the time derivative $d x / d r$. For some local system of coordinates $x_{j}$ the tangent vector in the r.h.s. of Eq. (5.1) adopts a form

$$
\dot{x}=\sum_{i k} \hat{\omega}_{M, i k} \frac{\partial H}{\partial x_{i}} \frac{\partial}{\partial x_{k}}
$$

which yields the following representation for Eq. (5.1):

$$
\frac{d x_{k}}{d \tau}=\sum_{i} \hat{\omega}_{M, i k}(x) \frac{\partial H(x)}{\partial x_{i}} .
$$

Equations of motion for the coupled dynamics are obtained in a straightforward way by substituting the Hamiltonian $H$ [Eqs. (4.1)-(4.5)] and the Poisson bracket $\hat{\omega}_{M}$ [Eqs. (4.15) and (4.16)] into Eq. (5.1). After some simple transformations they can be conveniently represented in terms of a system of coupled equations for the nuclear momenta $P_{\alpha}$ and for a point $y=(\mathbf{r}, \rho) \in G_{N}(E)$,

$$
\begin{aligned}
\frac{d P_{\alpha}}{d \tau}= & -\sum_{\nu} M_{\nu}^{-1} P_{\nu} \operatorname{Tr}\left\{\rho F_{\alpha \nu}(\mathbf{r})\right\}-\partial_{\alpha} u(\mathbf{r}) \\
& -\operatorname{Tr}\left\{\nabla_{\alpha} t(\mathbf{r}) \rho\right\}-\operatorname{Tr}\left\{\rho \nabla_{\alpha} V(\mathbf{r}) \rho\right\} \\
& +\partial_{\alpha} \mu_{N}(\mathbf{r}) \mathcal{E}(\tau)+\operatorname{Tr}\left\{\rho \nabla_{\alpha} \mu(\mathbf{r})\right\} \mathcal{E}(\tau) \\
\frac{d \mathbf{r}}{d \tau}= & \dot{\mathbf{r}} \\
\frac{d y}{d \tau}= & L_{y}(\mathbf{r})-i[t(\mathbf{r})+2 V(\mathbf{r}) \rho, \rho]+i \mathcal{E}(\tau)[\mu(\mathbf{r}), \rho]
\end{aligned}
$$


where $\dot{\mathbf{r}} \in \mathbb{T}_{\mathbf{r}}(M)$ is a tangent vector with the components $r_{\mu} \equiv M^{-1} \mu P \mu$. The combination of the last two terms in the r.h.s. of Eq. (5.6) constitutes an element of the tangent space to the fiber $G_{N}(V(\mathbf{r}))$.

Equations (5.4), (5.5), and (5.6) are represented using the connection $\nabla$ and have a clear geometrical interpretation. First, Eq. (5.5) represents the projection of Eq. (5.6) from $G_{N}(E)$ onto the configuration space $M_{R}$, rather than an independent equation, and is included into the system mostly for traditional reasons. The first term in Eq. (5.4) is induced by the intensity of the gauge field $F_{\mu \nu}(\mathbf{r})$ and is of purely geometrical origin. The sum of the rest terms has a natural interpretation: It represents the derivative of the effective potential energy which includes the electron energy with respect to the nuclear positions. The derivatives of $t(\mathbf{r}), \mu(\mathbf{r})$, and $V(\mathbf{r})$ which constitute sections of the bundles $\operatorname{End}(E)$ and End $(\operatorname{End}(E))$ are naturally taken using the corresponding connections induced by the connection $\nabla$ in E. In Eq. (5.6) the first term represents a shift along the connection which is determined by the velocity vector $\dot{\mathbf{r}}$ in the configuration space $M_{R}$. The other two terms represent the dynamics along the fiber, i.e., in the electronic space alone, and are given by the r.h.s. of a purely electronic TDHF equation [6].

Introducing a local basis set $v_{j}(\mathbf{r})$ in $E$, i.e., a basis set of single-electron orbitals, Eq. (5.6) can be decoupled into its projection onto $M_{R}$ [Eq. (5.5)] and an equation for the matrix elements $\rho_{i j}$ of $\rho$ defined by

$$
\rho v_{j}(\mathbf{r})=\sum_{i} \rho_{i j} v_{j}(\mathbf{r}) .
$$

Equation (5.6) adopts the form

$$
\begin{aligned}
\frac{d \rho}{d \tau}=\sum_{\nu}\left[A_{\nu}, \rho\right] \dot{r}_{v}-i[t(\mathbf{r})+ & 2 V(\mathbf{r}) \rho, \rho] \\
& +i \mathcal{E}(\tau)[\mu(\mathbf{r}), \rho] .
\end{aligned}
$$

The shift along the connection in Eq. (5.8) is represented by the coefficients $A_{v}$ of the connection $\nabla$ for the chosen basis set [Eq. (3.4)] or in other words by the gauge field. Equations of motion in some coordinate representation can be alternatively derived using the algebraic representation of the Poisson bracket [Eqs. (4.23), (4.24), (4.25), and (4.26)]. This will be done in the Appendix.

Summarizing, the input for the coupled electronnuclear dynamics consists of a fiber bundle $p: E \rightarrow$ $M_{R}$ over the configuration space $M_{R}$, a connection $\nabla$ in it, the functions $\mu(\mathbf{r})$ and $\mu_{N}(\mathbf{r})$, the sections $t(\mathbf{r})$ and $\mu(\mathbf{r})$ of $\operatorname{End}(E)$, and the section $V(\mathbf{r})$ of $\operatorname{End}(\operatorname{End}(E))$. They can be expressed in terms of the parameters of the original Hamiltonian by utilizing the immersion $E \subset M_{R} \times U$. However, once they are calculated any additional information on this immersion is irrelevant and the dynamics is expressed using the internal terms (i.e., the aforementioned input) only.

\section{Optical Response at Intermediate Frequencies: Contributions from Nuclear Motions}

In this section we derive equations of motion which provide an effective procedure for computing the polarizabilities in the intermediate frequency limit, i.e., when the optical frequency is higher than the vibrational frequencies but is still low compared to the frequencies of the electronic transitions. In this limit the deviation of the molecular geometry $\mathbf{r}$ induced by the driving field from its equilibrium position $\mathbf{r}=0$ is small. This implies that the optical response can be considered as a perturbation to the electronic contribution, as opposed to the purely static case. The calculations can be carried out as an expansion in the inverse nuclear mass $M^{-1}$. The first-order correction which is a subject of this section can be obtained by expanding the CEND equations [Eqs. (5.4), (5.5), (5.6)] to the first order in $M^{-1}$. This can be done as follows. In the zero order Eq. (5.5) yields $\mathbf{r} \equiv 0$. In Eq. (5.4) we omit the first term in the r.h.s and set $\mathbf{r}=0$ in the remaining terms. Because of $\mathbf{r}=0$ the electronic dynamics occurs in the space $G_{N}(V(0))$ and is represented by the motion of the density operator $\rho \in G_{N}(V(0))$. Setting $\mathbf{r}=0$ in Eq. (5.6) the first term in the r.h.s. vanishes and Eq. (5.6) adopts the form of the TDHF equations which describes the electronic dynamics for fixed nuclei. We now turn to the first order terms. To first order the deviation $\gamma$ of the actual position of $\left(\mathbf{r}, \rho^{\prime}\right) \in G_{N}(E)$ from its zero order position $(0, \rho)$ is described by a vector $\gamma \in \mathbb{T}_{(0, \rho)}\left(G_{N}(E)\right)$ which has a meaning of an infinitesimal derivation. Using the connection $\nabla$ the derivation $\gamma$ can be decomposed as

$$
\gamma=L_{(0, \rho)}(\mathbf{r})+\xi
$$

where $\mathbf{r} \in \mathbb{T}_{0}\left(M_{R}\right)$ is the nuclear component of the derivation and $\xi \in \mathbb{T}_{\rho} G_{N}\left(V^{(0)}\right)$ is the electronic component which is tangent to the fiber. $\xi$ is represented by an operator $\xi \in \operatorname{End}(V(0))$. We reiterate that $L_{(0, \rho)}(\mathbf{r}) \in \mathbb{T}_{(0, \rho)}\left(\nabla, G_{N}(E)\right)$. The first order in $M^{-1}$ terms in the nuclear momenta $P$ do not contribute to the optical response to given order (these contributions start with $\mathrm{M}^{-2}$ terms) and are not considered. 
The first order derivation is, therefore, represented in terms of the connection $\nabla$ which is an invariant object. The equation for $\mathbf{r}\left(d r_{\alpha} / d \tau\right)=M_{\alpha}^{-1} P_{\alpha}$ follows immediately from Eq. (5.5) and being combined with the zero order equation for $P$ yields the equation for $\mathbf{r}$. The equation for $\xi$ is obtained by expanding Eq. (5.8) to first order. Combining the three equations we obtain a closed coupled system of equations for $\rho, \mathbf{r}$, and $\xi$ which has the form

$$
\begin{aligned}
\frac{d P}{d \tau}= & -i[t+2 V \rho, \rho]+i \mathcal{E}(\tau)[\mu, \rho] \\
M_{\alpha} \frac{d^{2} r_{\alpha}}{d \tau^{2}}= & -u_{\alpha}+\operatorname{Tr}\left(t_{\alpha} \rho\right)-\operatorname{Tr}\left(\rho V_{\alpha} \rho\right) \\
& +\mu_{N, \alpha} \mathcal{E}(\tau)+\operatorname{Tr}\left(\mu_{\alpha} \rho\right) \mathcal{E}(\tau) \\
\frac{d \xi}{d \tau}= & -i[t+2 V \rho \xi]-i 2[V \xi, \rho]+i \mathcal{E}(\tau)[\mu, \xi] \\
& -i \sum_{\alpha}\left[t_{\alpha}+2 V_{\alpha} \rho, \rho\right] r_{\alpha} \\
& +i \mathcal{E}(\tau) \sum_{\alpha}\left[\mu_{\alpha}, \rho\right] r_{\alpha}
\end{aligned}
$$

The polarization $\mathcal{P}$ keeping the terms to first order in $M^{-1}$ has the form

$$
\begin{aligned}
\mathcal{P} & =\mathcal{P}^{(0)}+\mathcal{P}^{(1)} \\
\mathcal{P}^{(0)} & =\mu_{N}+\operatorname{Tr}(\mu \rho) \\
\mathcal{P}^{(1)} & =\sum_{\alpha} \mu_{N, \alpha} r_{\alpha}+\operatorname{Tr}(\mu \xi)+\sum_{\alpha} \operatorname{Tr}\left(\mu_{\alpha} \rho\right) r_{\alpha} .
\end{aligned}
$$

In Eqs. (6.2)-(6.4) and (6.5), (6.7) we used the notation $t \equiv t(0), V \equiv V(0)$, etc., and $\left.t_{\mu} \equiv \nabla_{\mu} t\right|_{\mathbf{r}=0}$, $\left.V_{\mu} \equiv \nabla_{\mu} V\right|_{\mathbf{r}=0}$, etc.

\section{Appendix A: Basic Structures}

In the appendix we summarize the basic structures and objects involved in our formalism using the language of differential geometry.

We denote by $M_{R}$ the nuclear configuration space whose points $\mathbf{r} \in M_{R}$ characterize the positions of the nuclei. The nuclear phase space $M_{0}$ is represented by the cotangent bundle $M_{0} \equiv \mathbb{T}^{*}\left(M_{R}\right)$ with the natural projection $p_{0}: M_{0} \rightarrow M_{R}$. The fiber over $\mathrm{r} \in M_{R}$ represents the momenta $P \in \mathbb{T}_{\mathrm{r}}^{*}\left(M_{R}\right)$ which can be considered as linear functionals acting on tangent vectors which belong to the tangent space $\mathbb{T}_{\mathbf{r}}\left(M_{R}\right)$ at point $\mathbf{r}$. The latter are represented by the fibers of the tangent bundle $\mathbb{T}\left(M_{R}\right)$ over $M_{R}$. The sections of the tangent bundle are vector fields which are represented by first order differential operators with a natural definition of the commutator.
$U$ denotes the space of all single-electron orbitals which is assumed to be finite dimensional $\operatorname{dim} U=N_{0}^{\prime}$ whereas $N_{0}^{\prime}$ can be very large. The classical electronic phase space $M_{1}$ of the extended system is given by the Grassmanian $M_{1} \equiv G_{N}(U)$ which represents the set of $N$-dimensional vector subspace of $E$. It can considered as a subspace $M_{1} \subset$ $\operatorname{End}(U)$ :

$$
\begin{array}{r}
G_{N}(U)=\left\{\rho \in \operatorname{End}(U) \mid \operatorname{rank} \rho=N, \rho^{2}=\rho,\right. \\
\left.\rho^{+}=\rho\right\} .
\end{array}
$$

The full classical phase of the coupled extended system of electrons and nuclei is represented by $M_{0} \times M_{1}$

The relevant single-electron orbitals are described by a vector bundle $p: E \rightarrow M_{R}$. Its fiber $V(\mathbf{r}) \equiv p^{-1}(\mathbf{r})$ at point $\mathbf{r}$ determines the relevant electron orbitals for the molecular geometry which corresponds to $\mathbf{r}$. All fibers have the same dimension $\operatorname{dim} V(\mathbf{r})=N_{0}$.

The bundle $G_{N}(p): G_{N}(E) \rightarrow M_{R}$ with the fibers $G_{N}(V(\mathbf{r}))$ represents the set of classical electronic phase spaces parametrized by the configuration space. Equation (A.1) applied to $V(\mathbf{r})$ instead of $U$ yields the immersion $G_{N}(V(\mathbf{r})) \subset \operatorname{End}(V(\mathbf{r}))$ which generates the immersion of bundles $G_{N}(E) \subset$ End $(E)$. This allows us to consider points in $G_{N}(E)$ as pairs $(\mathbf{r}, \rho)$ with $\mathbf{r} \in M_{R}$ and $\rho \in \operatorname{End}(V(\mathbf{r}))$.

The joint relevant phase space $M$ is given by the product of the cotangent bundle $M_{0}$ and $G_{N}(E)$,

$$
M \equiv M_{0} \times_{M_{R}} G_{N}(E),
$$

where the product of the bundles is defined in a standard way $[9-11]$ :

$$
\begin{aligned}
M_{0} \times_{M_{R}} G_{N}(E) \equiv\left\{(x, \gamma) \in M_{0} \times G_{N}(E)\right. & \left.\mid p_{0}(x)=G_{N}(p)(\gamma)\right\} .
\end{aligned}
$$

We have a natural immersion $M \subset M_{0} \times_{M_{R}} \operatorname{End}(E)$. The immersion $G_{N}(V(\mathbf{r})) \subset G_{N}(U)$ induces the immersion $M \subset M_{0} \times M_{1}$ of the relevant phase space into the phase space of the extended system.

\section{Appendix B: Connections in Bundles and Gauge Fields}

In this appendix we summarize the basic definitions and properties of connections in fiber bundles involved in our formulism. Let $\mathcal{A}_{0}(E)$ represent the sections of a vector bundle $E$ over $M_{R}$ and let $\mathcal{A}_{j}\left(M_{R}\right)$ represent the differential $j$-forms on $M_{R}$. We then denote by $\mathcal{A}_{j}(E) \equiv \mathcal{A}_{0}(E) \otimes \mathcal{A}_{j}\left(M_{R}\right)$ the $j$-forms with the 
values in the bundle $E$. A connection $\nabla$ is a linear map $\nabla: \mathcal{A}_{0}(E) \rightarrow \mathcal{A}_{1}(E)$ which satisfies the property

$$
\nabla(f s)=f \nabla s+s \otimes d f
$$

for any section $s \in \mathcal{A}_{0}(E)$ of $E$ and any function $f \in$ $\mathcal{A}_{0}\left(M_{R}\right)$ on $M_{R}$. Representing in a system of local coordinates on $M_{R}$,

$$
\nabla s=\sum_{\mu}\left(\nabla_{\mu} s\right) d r_{\mu}
$$

we obtain a set of coefficients $\nabla_{\mu} s \in \mathcal{A}_{0}(E)$ which constitutes sections of $E$ and represents the derivatives of the section $s$.

It follows from Eq. (B.1) that

$$
\left[\nabla_{\mu}, \nabla_{\nu}\right](f s)=f\left[\nabla_{\mu}, \nabla_{\nu}\right] s
$$

for any function $f$ and section s. Equation (B.3) implies that for any section $s$ the 2-form $\sum_{\mu \nu}\left[\nabla_{\mu}\right.$, $\left.\nabla_{\nu}\right] s d r_{\mu} \wedge d r_{\nu}$ can be represented in the form

$$
\begin{aligned}
\sum_{\mu \nu}\left[\nabla_{\mu}, \nabla_{\nu}\right] s(\mathbf{r}) d r_{\mu} \wedge d r_{\nu} & =-\sum_{\mu \nu} F_{\mu \nu}(\mathbf{r}) s(\mathbf{r}) d r_{\mu} \wedge d r_{\nu} \\
& =-F(\mathbf{r}) s(\mathbf{r})
\end{aligned}
$$

where $F_{\mu \nu} \in \mathcal{A}_{0}(\operatorname{End}(E))$ are the coefficients of the 2-form $F \in \mathcal{A}_{2}(\operatorname{End}(E))$ with the values in $(\operatorname{End}(E))$ known as the curvature of the connection $\nabla$.

A connection $\nabla$ in $E$ induces connections in vector bundles constructed out of $E$; e.g., the induced connection in the bundle $\operatorname{End}(E)$ is determined by the condition

$$
\nabla(\xi s)=(\nabla \xi) s+\xi(\nabla s)
$$

for $s \in \mathcal{A}_{0}(E), \xi \in \mathcal{A}_{0}(\operatorname{End}(E))$.

Using a local basis of sections $v_{j}(\mathbf{r}) \in \mathcal{A}_{0}(E)$ a connection is represented by a set of coefficients $A_{\mu}(\mathbf{r})$ [Eq. (3.4)] which can be viewed as a gauge field. First, $\nabla_{\mu} s$ is given by a "long derivative" [Eq. (3.6)] which constitutes the main concept of gauge fields. Second, substituting Eq. (3.6) into Eq. (B.4), i.e., representing Eq. (B.4) using the basis set $v_{j}$, we immediately obtain

$$
F_{\mu \nu}(\mathbf{r})=\partial_{\mu} A_{\nu}(\mathbf{r})-\partial_{\nu} A_{\mu}(\mathbf{r})-\left[A_{\mu}(\mathbf{r}), A_{\nu}(\mathbf{r})\right]
$$

which constitutes the expression for the intensity of a gauge field. Finally, transforming to a new basis set by $v_{i}^{\prime}=\sum g_{k i} v_{k}$ we obtain the coefficients $A_{v}^{\prime}$ of the connection $\nabla$ in the new basis set $v_{i}^{\prime}$ by using Eq. (3.4) and the property given by Eq. (3.3):

$$
A_{\mu}^{\prime}(\mathbf{r})=g(\mathbf{r})^{-1} \partial_{\mu} g(\mathbf{r})+g^{-1}(\mathbf{r}) A_{\mu}(\mathbf{r}) g(\mathbf{r}) .
$$

Equation (B.7) constitutes the expression for a gauge transformation for a non-abelian gauge field.
An alternative definition of a connection which can be extended to the case of nonvector bundles (i.e., bundles whose fibers are not vector spaces), as opposed to the definition as a rule of differentiation, identifies a connection $\nabla$ in $E$ as a set of vector subspaces $\mathbb{T}_{x}(\nabla, E) \subset \mathbb{T}_{x}(E)$ of the tangent spaces to $E$ with $\operatorname{dim} \mathbb{T}_{x}(\nabla, E)=N_{R}[9]$. We denote by $N_{R}$ the dimensionality of the configuration space. Therefore, it can be considered as a section $\mathbb{T}(\nabla)$ of a bundle $G_{N_{R}}(\mathbb{T}(E)$ ), the latter $E$ being the Grassmanian of the tangent bundle over $E$ [11]. This definition can be also reformulated in terms of a set of lifting maps $L_{(\mathbf{r}, \gamma)}: \mathbb{T}_{\mathbf{r}}\left(M_{R}\right) \rightarrow \mathbb{T}_{(\mathbf{r}, \gamma)}(E)$ [9] which are connected with $\mathbb{T}_{x}(\nabla, E)$ in the following way: $i m L_{(\mathbf{r}, \gamma)}=$ $\mathbb{T}_{(\mathbf{r}, \gamma)}(\nabla, E)$ and $p L(\xi)=\xi$ for any $\xi \in \mathbb{T}_{\mathbf{r}}\left(M_{R}\right)$. The set of lifting maps can be described by an injective linear morphism $L(\nabla): p^{*}\left(\mathbb{T}\left(M_{R}\right)\right) \rightarrow \mathbb{T}(E)$ of two vector bundles over $E$.

The equivalence of the two definitions of connection in the case of vector bundles is established by combining Eqs. (3.4), (3.6), and (3.7). The second definition of connection also allows one to introduce the curvature $F(\nabla)$ of the connection. The curvature $F(\nabla) \in \mathcal{A}_{0}\left(\operatorname{Hom}\left(p^{*}\left(\mathbb{T}\left(M_{R}\right) \otimes \mathbb{T}\left(M_{R}\right)\right), \mathbb{T}^{(f)}(E)\right)\right)$ can be considered as a morphism,

$$
F(\nabla):\left(p^{*}\left(\mathbb{T}\left(M_{R}\right) \otimes \mathbb{T}\left(M_{R}\right)\right)\right) \rightarrow \mathbb{T}^{(f)}(E),
$$

of vector bundles over $E$ [11] where $\mathbb{T}^{(f)}(E) \subset \mathbb{T}(E)$ is represented by the vectors tangent to the fibers. $F(\nabla)$ is defined as follows. Starting with two vector fields $\xi, \gamma$ [which are sections of $\mathbb{T}\left(M_{R}\right)$ ] we lift them to the bundle by means of the lifting map $L(\nabla)$ which yields two vector fields $L(\xi)$ and $L(\gamma)$ in $E$ which lie in $\mathbb{T}(\nabla, E) . F(\xi \otimes \gamma)$ is obtained by projecting the commutator $[L(\xi), L(\gamma)]$ into the tangent spaces to the fibers,

$$
F(\xi \otimes \gamma) \equiv: P^{(f)}[L(\xi), L(\gamma)],
$$

where $P^{(f)}$ denotes the aforementioned projection.

The connection between the two definitions of curvature [Eqs. (B.4) and (B.9)] for vector bundles is easily established by choosing a local basis $v_{j}(\mathbf{r})$ and using the expression of Eq. (3.5). Equation (3.7) yields

$$
L\left(\frac{\partial}{\partial r_{\mu}}\right)=\frac{\partial}{\partial r_{\mu}}+\sum_{i k} A_{i k, \mu}(\mathbf{r}) s_{i} \frac{\partial}{\partial s_{k}} .
$$

A straightforward calculation yields, upon the substitution of Eq. (B.10) into Eq. (B.9),

$$
F\left(\frac{\partial}{\partial r_{\mu}} \otimes \frac{\partial}{\partial r_{\nu}}\right)=\sum_{i k} F_{i k, \mu \nu}(\mathbf{r}) s_{i} \frac{\partial}{\partial s_{k}} .
$$


Equation (B.11) implies that the tangent to the fiber vector at point $(\mathbf{r}, s)$ which gives the value of $F$ is $F_{\mu \nu}(\mathbf{r}) s$.

At the end of Section III we introduced a connection in $E$ induced by the immersion $E \subset M_{R} \times U$. It follows immediately from its definition that the coefficients of $\nabla$ in the basis set $v_{j}(\mathbf{r})$ are given by

$$
A_{i j, \mu}(\mathbf{r})=-\left\langle v_{j}(\mathbf{r}) \mid \partial_{\mu} v_{j}(\mathbf{r})\right\rangle .
$$

In Eq. (B.12) the sections $v_{j}(\mathbf{r})$ are considered as functions on $M_{R}$ valued in $U$. This connection is obviously compatible with the scalar product; i.e.,

$$
\partial_{\mu}\langle v(\mathbf{r}) \mid s(\mathbf{r})\rangle=\left\langle\nabla_{\mu} v(\mathbf{r}) \mid s(\mathbf{r})\right\rangle+\langle v(\mathbf{r}) \mid \nabla s(\mathbf{r})\rangle
$$

for any two sections $v(\mathbf{r}), s(\mathbf{r})$ of $E$. The space $U$ has also a real structure; i.e., one can complex conjugate the states. In the absence of magnetic field the relevant orbitals can be chosen to be real which implies that the connection $\nabla$ is compatible with the real structure. This is a signature of the time-reversal symmetry. The connection $\nabla$ therefore becomes an orthogonal rather than a unitary connection.

Finally, we note that the expression for the nuclear part $\omega_{M}^{(0)}$ of the symplectic structure [Eq. (4.13)] can be derived in a straightforward way by extending the basis set $v_{j}(\mathbf{r})$ in $V(\mathbf{r})$ to complete basis set in $U$ with further use of Eqs. (4.6)-(4.8) together with Eq. (B.12). However, the equivalence of the two definitions of the connection [Eqs. (B.4) and (B.9)] established by Eq. (B.11) makes even this simple calculation unnecessary. The first line in Eq. (4.13) is obvious. The second relation is established by simply comparing Eqs. (4.8) and (B.9). In both cases we take two vector fields which belong to the spaces $\mathbb{T}_{x}(\nabla)$, commute them, and take the trace of the product of $\rho$ with the commutator. Equation (B.11) immediately yields Eq. (4.13).

In the case when the time-reversal symmetry is not broken and the connection $\nabla$ is orthogonal we have $\operatorname{Tr}\left(\rho F_{\mu \nu}(\mathbf{r})\right) \equiv 0$ and the first term in Eq. (5.4) which represents the Lorentz force naturally vanishes.

\section{ACKNOWLEDGMENTS}

The support of the National Science Foundation is gratefully acknowledged.

\section{References}

1. Blaizot, J.-P.; Ripka, G. Quantum Theory of Finite Systems; The MIT Press: Cambridge, MA, 1986; Thouless, D. J. Nucl Phys 1960, 21, 225.

2. Chernyak, V.; Mukamel, S. J Chem Phys 2000, 112, 3572; Chernyak, V.; Mukamel, S. J Chem Phys 1996, 104, 444.

3. Chernyak, V.; Schulz, M.; Mukamel, S.; Tretiak, S.; Tsiper, E. J Chem Phys 2000, 113, 36.

4. Tretiak, S.; Chernyak, V.; Mukamel, S. J Chem Phys 2000, 112, 3572; Tretiak, S.; Chernyak, V.; Mukamel, S. JACS 1997, 119, 11408.

5. Deumens, E.; Diz, A. D.; Longo, R.; Öhrn, Y. Rev Mod Phys $1988,109,4156$

6. Chernyak, V.; Mukamel, S. J Phys Chem A 2000, 104, 4263.

7. Davidovich, L.; Landau, L.; Lifshitz, E. M. Mechanics; Butterworth-Heinemann, 1976.

8. Vogtmann, K.; Weinstein, A.; Arnold, V. I. Mathematical Methods in Classical Mechanics; Springer-Verlag: New York, 1978.

9. Kramer, P.; Saraceno, M. Geometry of the Time-Dependent Variational Principle; Springer: New York, 1981.

10. Kirillov, A. A. Elements of the Theory of Representations; Springer: Berlin, 1976.

11. Klander, J. R.; Skagerstam, B.-S. Coherent States, Applications in Physics and Mathematical Physics; World Scientific: Singapore, 1985.

12. Perelomov, A. M. Generalized Coherent States and Their Applications; Springer: New York, 1986.

13. Kobayashi, S.; Nomizu, K. Foundations of Differential Geometry; Wiley: New York, 1996.

14. LePotier, J. Lectures on Vector Bundles; Cambridge Univ. Press: New York, 1997.

15. Manin, Y. I.; Koblitz, N.; King, J. R. Gauge Field Theory and Complex Geometry; Springer-Verlag: Berlin, 1988.

16. Griffiths, P.; Harris, J. Principles of Algebraic Geometry; Wiley: New York, 1994.

17. Arnold, V. I. Ordinary Differential Equations; MIT Press: Cambridge, 1973; Arnold, V. I. Geometrical Methods in the Theory of Ordinary Differential Equations; Springer-Verlag: New York, 1988. 